\title{
Axion mass prediction from minimal grand unification
}

\author{
Luca Di Luzio, ${ }^{1, *}$ Andreas Ringwald, ${ }^{2, \dagger}$ and Carlos Tamarit ${ }^{3, \$}$ \\ ${ }^{1}$ Institute for Particle Physics Phenomenology, Department of Physics, Durham University, \\ DH1 3LE Durham, United Kingdom \\ ${ }^{2}$ Deutsches Elektronen-Synchrotron DESY, Notkestraße 85, D-22607 Hamburg, Germany \\ ${ }^{3}$ Physik Department T70, Technische Universität München, \\ James Franck Straße 1, 85748 Garching, Germany
}

(Received 6 August 2018; published 12 November 2018)

\begin{abstract}
We propose a minimal realization of the Peccei-Quinn mechanism in a realistic SU(5) model, where the axion mass is directly connected to the grand unification scale. Accounting for constraints from proton decay, collider searches and gauge coupling unification, we predict the axion mass $m_{a} \in[4.8,6.6] \mathrm{neV}$. The upper bound relaxes to $m_{a}<330 \mathrm{neV}$ for a tuned flavor structure of the proton decay operators. The predicted mass window will be complementarily probed by the axion dark matter experiments ABRACADABRA and CASPER-Electric, which could provide indirect evidence for the scale of grand unification before the observation of proton decay.
\end{abstract}

DOI: 10.1103/PhysRevD.98.095011

\section{INTRODUCTION}

It is a widespread belief that the standard model (SM) of particle physics should break down at some intermediate energy between the electroweak and the Planck scale. The quantum numbers of the SM fermions, together with the gauge coupling evolution at high energies, hint at a unified gauge dynamics around $10^{15} \mathrm{GeV}$. This scale is generically compatible with constraints from the nonobservation of proton decay, the smoking-gun signature of grand unified theories (GUTs). The search for proton decay was vigorously pushed in the past decades, and has reached its limits with the Super-Kamiokande (SK) observatory [1]. Planned largevolume facilities, such as Hyper-Kamiokande (HK) [2], will improve the bound on the proton lifetime by 1 order of magnitude in the next decade. Though fundamentally important, that translates only into a factor of 2 on the GUT scale.

Another well-motivated framework which points to intermediate mass scales is associated with the Peccei-Quinn (PQ) solution of the strong $C P$ problem [3,4], which predicts the axion as a low-energy remnant $[5,6]$. The axion needs to be extremely light and decoupled, and in a certain mass range is also a viable dark matter (DM) candidate [7-9]. The experimental program for axion searches is rapidly

\footnotetext{
*luca.di-luzio@durham.ac.uk †andreas.ringwald@desy.de

carlos.tamarit@tum.de
}

Published by the American Physical Society under the terms of the Creative Commons Attribution 4.0 International license. Further distribution of this work must maintain attribution to the author(s) and the published article's title, journal citation, and DOI. Funded by SCOAP. evolving and a large portion of the parameter space predicted by the QCD axion will be probed in the next decade [10]. From an experimental point of view, however, one of the main bottlenecks of axion DM searches (e.g., those exploiting microwave cavities or nuclear magnetic resonance techniques) is the need to perform a fine scan in the axion mass in order to meet a resonance condition. Since the axion mass is not predicted by the PQ mechanism, any extra theoretical information which could pin-down the axion mass precisely would be extremely helpful for experiments.

Following recent attempts to revive PQ-GUTs in $\mathrm{SO}(10)$ [11] (see also [12-18]), in this paper we revisit the more minimal option of SU(5). The simplest implementation of the axion in nonsupersymmetric ${ }^{1} \mathrm{SU}(5)$ was proposed by Wise, Georgi and Glashow (WGG) [22] (see also [23]). However, similarly to the original SU(5) model of Georgi and Glashow (GG) [24], the WGG model is ruled out because of gauge coupling unification and neutrino masses. An elegant and minimal way to fix both these issues in the GG model was put forth by Bajc and Senjanović [25], which add to the minimal GG field content a single Majorana fermion representation, $24_{F}$, in the adjoint of $\mathrm{SU}(5)$. The extra degrees of freedom have the right quantum numbers to generate neutrino masses via a hybrid Type-I+III seesaw mechanism and ensure a proper

\footnotetext{
${ }^{1}$ The reader might wonder why we care for the fine-tuning of $\left|\theta_{\mathrm{QCD}}\right| \lesssim 10^{-10}$ and not for the electroweak-GUT hierarchy. A possible answer is that the strong $C P$ problem is qualitatively different from the hierarchy problem, and it is conceivable that the solution of the latter does not rely on a stabilizing symmetry (an interesting example is the possibility that a light Higgs might be selected by the cosmological evolution of the Universe [19-21]).
} 
unification pattern. In particular, the main observable emerging from detailed renormalization group analyses [25-27] is a clean correlation between light electroweak triplet states [constrained by the Large Hadron Collider (LHC)] and the unification scale (constrained by SK).

Having in mind the possibility of narrowing the axion mass range within a minimal and realistic extension of the WGG model, we extend the latter with a $24_{F}$, in analogy to the $\mathrm{GG}+24_{F}$ case. From the $\mathrm{GG}+24_{F}$ point of view, the PQ extension is also motivated by the lack of a DM candidate. Within the WGG model (or other realistic extensions) the axion mass can be put in one-to-one correspondence with the proton decay rate. This allows us to extract an upper bound on the axion mass. Including also the detailed information from gauge coupling unification available in the WGG $+24_{F}$ model, we are also able to set a lower bound on the axion mass from the nonobservation of electroweak-triplet states at LHC, thus predicting the following axion mass: $m_{a} \in[4.8,6.6] \mathrm{neV}$, where the upper bound holds in the absence of tuning of fermionic mixing. Furnished with this result, we provide the axion coupling to the SM fields and estimate the sensitivity of future axion DM experiments such as ABRACADABRA [28] and CASPEr $[29,30]$ in the relevant mass window.

\section{THE WGG MODEL}

Let us recall the main features of the WGG model [22]. While the fermion content is that of the original GG SU(5) [24], namely three copies of $\overline{5}_{F}$ and $10_{F}$ comprising the chiral SM matter fields, the scalar sector is extended to include a complex $24_{H}$ and two fundamentals, $5_{H}$ and $5_{H}^{\prime}$. The WGG Yukawa Lagrangian is, schematically, ${ }^{2}$

$$
\mathcal{L}_{Y}=\overline{5}_{F} 10_{F} 5_{H}^{* *}+10_{F} 10_{F} 5_{H},
$$

while the scalar potential (which we do not report here entirely) contains two nontrivial invariants which are affected by global re-phasings:

$$
V_{H} \supset 5_{H}^{\prime \dagger} 24_{H}^{2} 5_{H}+5_{H}^{\prime \dagger} 5_{H} \operatorname{Tr}\left(24_{H}^{2}\right) \text {. }
$$

Note that this structure resembles that of the DFSZ model [31,32]. In fact, the WGG Lagrangian is invariant under the global $\mathrm{U}(1)_{\mathrm{PQ}}$ transformation: $\overline{5}_{F} \rightarrow e^{-i \alpha / 2} \overline{5}_{F}$, $10_{F} \rightarrow e^{-i \alpha / 2} 10_{F}, 5_{H} \rightarrow e^{i \alpha} 5_{H}, 5_{H}^{\prime} \rightarrow e^{-i \alpha} 5_{H}^{\prime}$ and $24_{H} \rightarrow$ $e^{-i \alpha} 24_{H}$.

We have performed the minimization of the full scalar potential in [22] and computed the particle spectrum. In particular, it can be shown that the vacuum expectation value (VEV) configuration

$$
\left\langle 24_{H}\right\rangle=V \frac{1}{\sqrt{30}} \operatorname{diag}(2,2,2,-3,-3),
$$

\footnotetext{
${ }^{2}$ Nonrenormalizable operators or extra scalar representations are further required in order to correct the ratio between the masses of down quarks and charged leptons.
}

breaks $\mathrm{SU}(5) \times \mathrm{U}(1)_{\mathrm{PQ}}$ down to the $\mathrm{SM}$ gauge group with a single order parameter $V$. $^{3}$ The axion, the (pseudo) Nambu-Goldstone boson of the global $\mathrm{U}(1)_{\mathrm{PQ}}$, is dominantly contained in the phase along the SM singlet direction of $24_{H}$, i.e.,

$$
24_{H} \supset\left\langle 24_{H}\right\rangle \frac{1}{\sqrt{2}} e^{i a / V} .
$$

A crucial point of the WGG model is that the mass of the heavy vector leptoquark $V_{\mu}=(3,2,-5 / 6)$ mediating proton decay,

$$
m_{V}=\sqrt{\frac{5}{6}} g_{5} V,
$$

(where $g_{5}$ denotes the $\mathrm{SU}(5)$ gauge coupling) is directly connected to the axion decay constant ${ }^{4}$

$$
f_{a}=V / \hat{N},
$$

where $\hat{N}$ is the $\mathrm{U}(1)_{\mathrm{PQ}} \mathrm{SU}(3)_{C}-\mathrm{SU}(3)_{C}$ anomaly coefficient, e.g., $\hat{N}=6$ in the WGG model. This implies a direct connection between the axion mass and the proton decay rate.

By means of chiral Lagrangian techniques, we can recast the formula for the proton decay mode $p \rightarrow \pi^{0} e^{+}$in $\mathrm{SU}(5)$ as $[34,35]$ :

$$
\begin{aligned}
\Gamma_{p \rightarrow \pi^{0} e^{+}}= & \frac{m_{p}}{16 \pi f_{\pi}^{2}} A_{L}^{2}|\alpha|^{2}(1+D+F)^{2} \\
& \times\left(\frac{g_{5}^{2}}{2 m_{V}^{2}}\right)^{2}\left[4 A_{S L}^{2}+A_{S R}^{2}\right],
\end{aligned}
$$

where we have set unknown fermion mixing matrices equal to the identity. $A_{L}=1.25$ encodes the renormalization from the electroweak scale to the proton mass, $m_{p}=938.3 \mathrm{MeV} ; f_{\pi}=139 \mathrm{MeV}, D=0.81, F=0.44$ and $\alpha=-0.011 \mathrm{GeV}^{3}$ are phenomenological parameters given by the chiral Lagrangian and the lattice. $A_{S L(R)}$ are $O(1)$ short-distance renormalization factors from the GUT to the electroweak scale which depend on the intermediate-scale thresholds [36-38]. For example, $A_{S L(R)}=2.4(2.2)$, running within the SM up to $10^{15} \mathrm{GeV}$.

By using Eqs. (5) and (6) and the relation $m_{a}=$ $5.7 \mathrm{neV}\left(10^{15} \mathrm{GeV} / f_{a}\right)[39,40]$, we can reexpress Eq. (7) in the following parametric form:

$$
\begin{aligned}
\Gamma_{p \rightarrow \pi^{0} e^{+}} \simeq & \left(1.6 \times 10^{34} \mathrm{yr}\right)^{-1}\left(\frac{m_{a}}{3.7 \mathrm{neV}}\right)^{4}\left(\frac{6}{\hat{N}}\right)^{4} \\
& \times\left[0.83\left(\frac{A_{S L}}{2.4}\right)^{2}+0.17\left(\frac{A_{S R}}{2.2}\right)^{2}\right],
\end{aligned}
$$

\footnotetext{
${ }^{3}$ The recent work [33], bearing analogies with our proposal, crucially differs in the fact that the PQ symmetry is broken by an $\mathrm{SU}(5)$ singlet.

${ }^{4}$ We neglect corrections depending on weak-scale VEVs. For practical recipes on how to compute axion properties in GUTs, see [11].
} 
where we have highlighted in the first parenthesis the current proton decay bound from SK [1].

\section{AXION MASS PREDICTION IN WGG $+24_{F}$}

The extension of WGG with an extra $24_{F}$ is analogous to the proposal in [25]. Here, we highlight the main differences due to the presence of the PQ symmetry. The Yukawa Lagrangian is extended by

$$
\Delta \mathcal{L}_{Y}=\overline{5}_{F} 24_{F} 5_{H}+\operatorname{Tr} 24_{F}^{2} 24_{H}^{*} .
$$

The first term provides a Dirac Yukawa interaction for the triplet and singlet fields contained in $24_{F}$, while the second term generates a Majorana mass for the full multiplet upon SU(5) symmetry breaking. We leave implicit the presence of extra nonrenormalizable operators, which are needed to avoid a rank-one light neutrino mass matrix, and to split the mass of the $24_{F}$ submultiplets (see [25-27]). Equation (9) also fixes the PQ transformation of the new field: $24_{F} \rightarrow$ $e^{-i \alpha / 2} 24_{F}$; the total $\mathrm{U}(1)_{\mathrm{PQ}} \mathrm{SU}(3)_{C}-\mathrm{SU}(3)_{C}$ anomaly gives $\hat{N}=11$.

The possibility of narrowing down the axion mass range follows directly from unification constraints. The main obstacle for unification in the SM is the early convergence of the electroweak gauge couplings, $\alpha_{1}$ and $\alpha_{2}$, around $10^{13} \mathrm{GeV}$, at odds with proton decay bounds. Hence, the key ingredients for a viable unification pattern are additional particles charged under $\mathrm{SU}(2)_{L}$ which can delay the meeting of $\alpha_{1}$ and $\alpha_{2}$. Such a role in the WGG $+24_{F}$ model can be played by the electroweak fermion $T_{F}=(1,3,0)$ and scalar $T_{H}=(1,3,0)$ triplets contained in the $24_{F, H}$. They are predicted to be at the $\mathrm{TeV}$ scale, so that a large enough unification scale can be achieved.

Both types of triplets, if light enough, can give interesting signatures at the LHC. The fermionic component leads to same sign di-lepton events which violate lepton number [41]. A recent CMS analysis [42] sets a 95\% CL exclusion at $840 \mathrm{GeV}$, while projected limits at the High Luminosity LHC (HL-LHC) [43,44] give $m_{T_{F}} \gtrsim 2 \mathrm{TeV}$. Bosonic triplets can affect the di-photon Higgs signal strength, but the bound is milder compared to the fermionic triplet and model dependent [45]. Here, we conservatively assume $m_{T_{H}} \gtrsim 200 \mathrm{GeV}$.

The complete unification pattern including also the convergence of $\alpha_{3}$ with $\alpha_{1}$ and $\alpha_{2}$ requires heavier colored particles. These are the color-octet fermions and scalars contained in the $24_{F, H}$, whose masses are required to be around $10^{8} \mathrm{GeV}$, well beyond the LHC energy range.

The main prediction of gauge coupling unification is hence a clean correlation between a triplet mass parameter

\footnotetext{
${ }^{5}$ Compared to the $\mathrm{GG}+24_{F}$ case we have in principle extra thresholds due to fact that the $24_{H}$ is complex. However, the constraints coming from the minimization of the scalar potential allow only one real triplet to be light, otherwise a colored octet would be lowered to the triplet mass scale, spoiling nucleosynthesis [25].
}

$m_{3}=\left(m_{T_{F}}^{4} m_{T_{H}}\right)^{1 / 5}$ and the unification scale. The latter is operatively defined as the energy scale where $\alpha_{1}$ and $\alpha_{2}$ meet up to GUT-scale thresholds [46,47], and it can be identified with $m_{V}$, the mass of the heavy vector leptoquark $V_{\mu}$ mediating proton decay. Thanks to Eqs. (5) and (6), we can trade $m_{V}$ for the axion mass, which allows us to present the unification constraints in the $\left(m_{a}, m_{3}\right)$ plane.

Following Ref. [27], we have performed a gauge coupling unification analysis including the leading NNLO corrections coming from the 2-loop matching coefficients and the 3-loop beta functions due to the fermion and scalar triplets. The extra thresholds affecting the evolution of $\alpha_{1}$ and $\alpha_{2}$ are fixed in such a way that the value of $m_{3}$ is maximized (cf. [27] for more details), which defines the parameter $m_{3}^{\max }$. The results are displayed in Fig. 1, which shows the correlation in the $\left(m_{a}, m_{3}^{\max }\right)$ plane. Taking into account the present bounds from LHC (on both fermion and scalar triplets) and SK (obtained by setting $A_{S L}=2.6$ and $A_{S R}=2.4$ in Eq. (8), which follow from the unification analysis), the preferred axion mass window is

$$
m_{a} \in[4.8,6.6] \mathrm{neV} .
$$

Future projections at HL-LHC (on the fermion triplet) and HK (10 years data taking [2]) provide complementary tests of this scenario.

Allowing for cancellations in the flavor structure of proton decay operators, the bound can be relaxed by more than 1 order of magnitude $[48,49]$. For heavy Majorana neutrinos, the weakest constraint arises when all the main decay channels are tuned away, except those involving strange mesons (which due to unitarity cannot be set to zero). The results in [48], updated with the latest experimental limit $\tau / \mathcal{B}\left(p \rightarrow K^{0} \mu^{+}\right)>1.3 \times 10^{33}$ yr [50], translate into the absolute upper bound $m_{a}<330 \mathrm{neV}$. Similarly, from the projections at HK [2] in the $p \rightarrow K^{+} \bar{\nu}$ channel we estimate $m_{a}<160 \mathrm{neV}$.

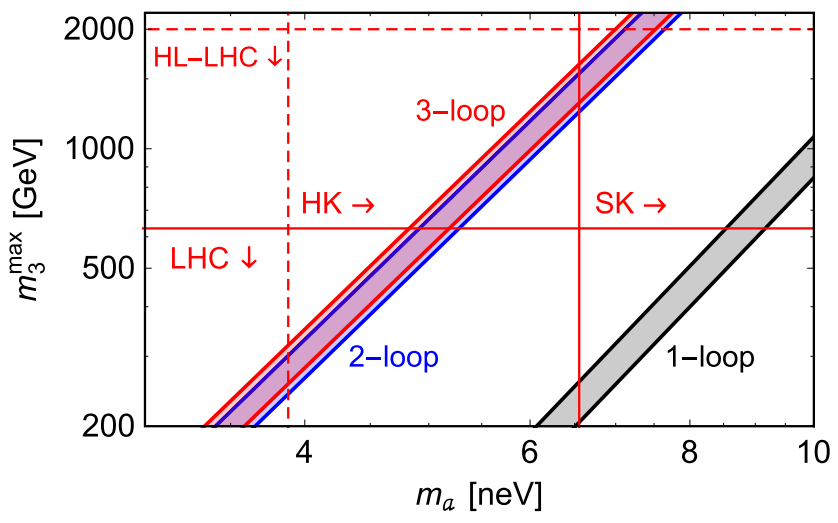

FIG. 1. Maximal triplet mass parameter as a function of the axion mass. Grey, blue and red bands denote, respectively, the correlation at 1,2 and 3 loops (shaded regions encode the $1 \sigma$ uncertainty on the electroweak gauge couplings). The full horizontal (vertical) red line is the current exclusion from LHC (SK), the dashed horizontal (vertical) red line is the projected exclusion from HL-LHC (HK). 


\section{SENSITIVITY OF FUTURE AXION DM SEARCHES}

An axion in this mass range is extremely weakly coupled to SM particles, since its couplings to e.g., photons $(\gamma)$, electrons $(e)$, protons $(p)$, and neutrons $(n)$ are inversely proportional to the axion decay constant,

$$
\mathcal{L}_{a} \supset \frac{\alpha}{8 \pi} \frac{C_{a \gamma}}{f_{a}} a F_{\mu \nu} \tilde{F}^{\mu \nu}-\frac{1}{2} \frac{C_{a f}}{f_{a}} \partial_{\mu} a \bar{\Psi}_{f} \gamma^{\mu} \gamma_{5} \Psi_{f} .
$$

while the coefficients $C_{a x}$ are of order unity. In the WGG + $24_{F}$ model, we find

$$
\begin{aligned}
& C_{a \gamma}=\frac{8}{3}-1.92(4), \quad C_{a e}=\frac{2}{11} \sin ^{2} \beta, \\
& C_{a p}=-0.47(3)+\frac{6}{11}\left[0.288 \cos ^{2} \beta-0.146 \sin ^{2} \beta \pm 0.02\right], \\
& C_{a n}=-0.02(3)+\frac{6}{11}\left[0.278 \sin ^{2} \beta-0.135 \cos ^{2} \beta \pm 0.02\right],
\end{aligned}
$$

where we introduced the ratio of the electroweak VEVs, $\tan \beta=\left\langle 5_{H}\right\rangle /\left\langle 5_{H^{\prime}}\right\rangle$. This makes the GUT axion clearly invisible for purely laboratory based experiments.

However, axions in this mass range are known to be excellent DM candidates [7-9] which can be searched for in axion DM direct detection experiments. Very light axion DM tends to be overproduced, and the measured amount of cold DM can only be explained if the PQ symmetry remained broken during and after inflation. ${ }^{6}$ In this case, the axion's relic abundance depends both on the mass and on the initial value of the axion field $a_{i}$ in units of the decay constant, $\theta_{i}=a_{i} / f_{a}$, inside the causally connected region which is inflated into our visible universe, cf. [40,51]:

$$
\Omega_{a} h^{2}=0.12\left(\frac{5.0 \mathrm{neV}}{m_{a}}\right)^{1.165}\left(\frac{\theta_{\mathrm{i}}}{1.6 \times 10^{-2}}\right)^{2} .
$$

Thus an axion in the neV mass range can make $100 \%$ of $\mathrm{DM}$, if the initial field value $\theta_{i}$ is of order $10^{-2}{ }^{7}$ In this cosmological scenario, however, isocurvature quantum fluctuations of a massless axion field during inflation may leave an imprint in the temperature fluctuations of the cosmic microwave background (CMB) [53,54], whose amplitude is stringently constrained by observations. In the case that the $24_{H}$ stays at a broken minimum of the potential throughout inflation (e.g., for a SM-singlet inflaton), those constraints translate in an upper bound on the Hubble expansion rate during inflation [55-57]:

\footnotetext{
${ }^{6}$ This solves at the same time the cosmological SU(5) monopole problem and the PQ domain-wall problem (the WGG $+24_{F}$ model has domain-wall number 11).

${ }^{7}$ This value can be supported by anthropic arguments [52].
}

$$
H_{I}<5.7 \times 10^{8} \mathrm{GeV}\left(\frac{5.0 \mathrm{neV}}{m_{a}}\right)^{0.4175} .
$$

Intriguingly, these isocurvature constraints can disappear completely in the case of nonminimal chaotic inflation [58-60] along one of the components of the $24_{H}$. In this case, during inflation the $24_{H}$ is not at a minimum, Goldstone's theorem does not apply, and the lightest fluctuations orthogonal to the inflaton can have masses above $H_{I}$ as long as the parameter $\xi_{24_{H}}$, describing the nonminimal coupling to the Ricci scalar, $S \supset-\int d^{4} x \sqrt{-g} \xi_{24_{H}} \operatorname{Tr}\left(24_{H}^{2}\right) R$, is larger than $\sim 0.01$. For $\xi_{24_{H}}$ above this value, the power spectra of the isocurvature fluctuations become exponentially suppressed and the CMB bounds can be avoided. In such scenarios, one still needs to ensure that the PQ symmetry is never restored after inflation; we expect that this might be possible for small enough quartic and Yukawa couplings of the $24_{H}$, but a dedicated analysis generalizing the reheating calculations in Ref. [51] is needed.

The DM experiment ABRACADABRA [28] has very good prospects to probe the axion photon coupling, $g_{a \gamma}=$ $\alpha C_{a \gamma} /\left(2 \pi f_{a}\right)$, in the relevant mass region. This is shown in Fig. 2, from which we infer that the whole parameter space of the WGG $+24_{F}$ model (including the tuned region) can be tested in the late stages of the experiment.

In Fig. 3, we show the projected sensitivity of CASPErElectric [29,30], which aims to search for oscillating nucleon electric dipole moments $(\mathrm{EDM}) d_{n}(t)=$ $g_{a D} \frac{\sqrt{2 \rho_{\mathrm{DM}}}}{m_{a}} \cos \left(m_{a} t\right)$ [61], where $g_{a D}$ is the model-independent coupling of the axion to the nucleon EDM operator, $\mathcal{L}_{a} \supset-\frac{i}{2} g_{a D} a \bar{\Psi}_{N} \sigma_{\mu \nu} \gamma_{5} \Psi_{N} F^{\mu \nu}$, and $\rho_{\mathrm{DM}}=0.3 \mathrm{GeV} / \mathrm{cm}^{3}$ is the local energy density of axion DM. The QCD axion band in Fig. 3 indicates the theoretical uncertainty of the

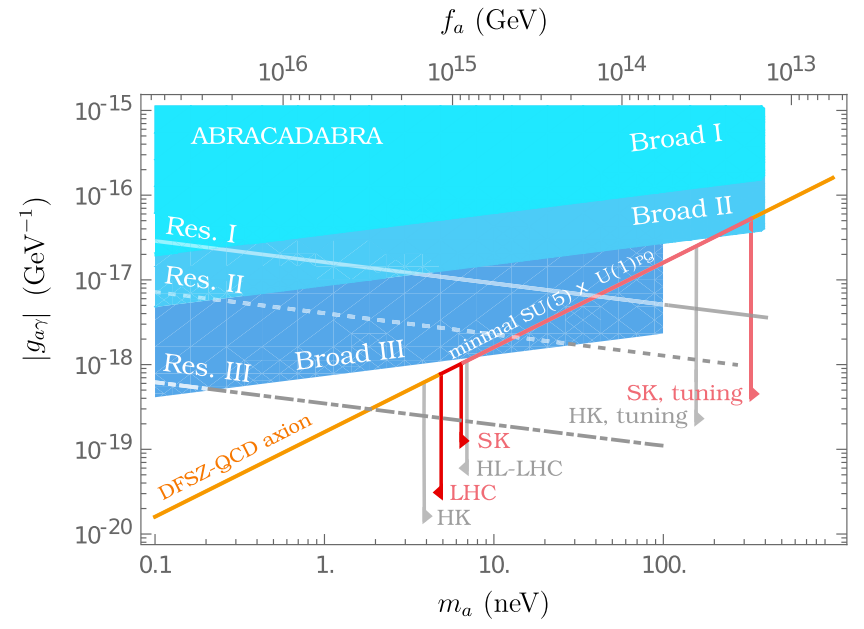

FIG. 2. Axion coupling to photons, $g_{a \gamma}$, versus axion mass $m_{a}$. The blue regions give the projected sensitivities of broadband ("Broad") and resonant ("Res.") search modes of ABRACADABRA from Ref. [28]. 


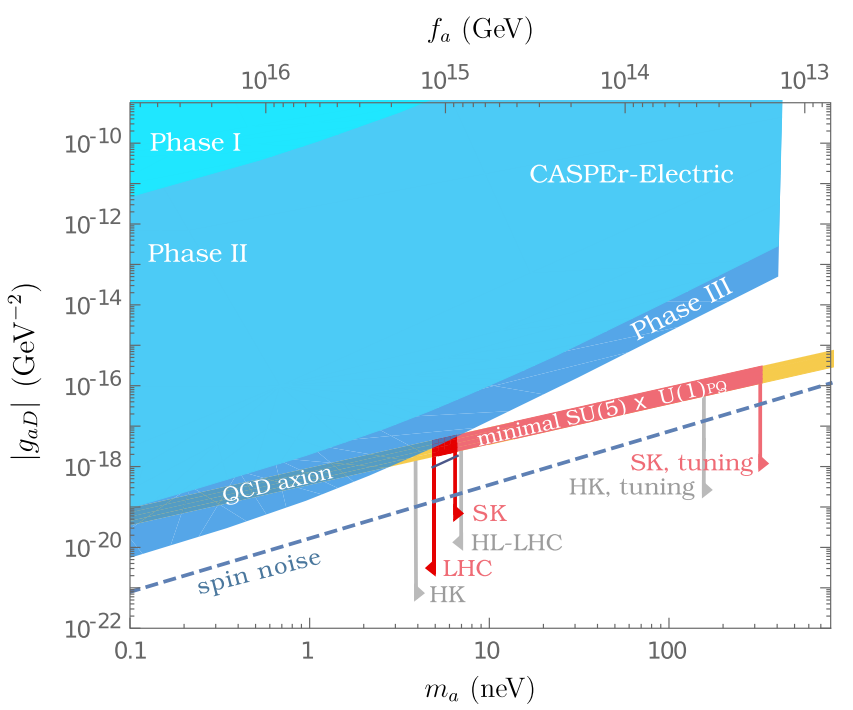

FIG. 3. Axion coupling to the nucleon EDM operator, $g_{a D}$, versus axion mass $m_{a}$. The blue regions give the projected sensitivities of CASPEr-Electric from Ref. [30]. The short, full blue line reflects a factor of 3 improvement in sensitivity for a search just concentrated on the preferred mass region.

nonperturbative estimate of $g_{a D}$ from [62]. The preferred axion mass window (10) could be probed in phase III of CASPEr-Electric. In fact, the sensitivity in $g_{a D}$ improves with the scanning time as $t^{1 / 4}$. This amounts to a factor of 3 improvement (denoted by a short, full blue line in Fig. 3), if CASPEr-Electric spends all the measurement time just on the preferred mass region.

On the other hand, the projected sensitivity of CASPErWind [30], which exploits the axion nucleon coupling $g_{a N}=C_{a N} /\left(2 f_{a}\right)(N=p, n)$ to search for the axion DM wind due to the movement of the Earth through the Galactic DM halo [61], misses the preferred coupling vs mass region by 2 orders of magnitude or more, even in its phase II. We show this in Fig. 4, where the theoretical uncertainty of the axion band is obtained from the errors in the coefficients of Eq. (12), and from varying $\tan \beta \in[0.28,140]$ in the perturbative unitarity domain [63].

\section{CONCLUSIONS}

In this paper we have proposed a minimal implementation of the PQ mechanism in a realistic SU(5) model, which predicts a narrow axion mass window [cf. Eq. (10)] which can be directly tested at future axion DM experiments and indirectly probed by collider and proton decay experiments. In principle, a precise determination of $m_{a}$

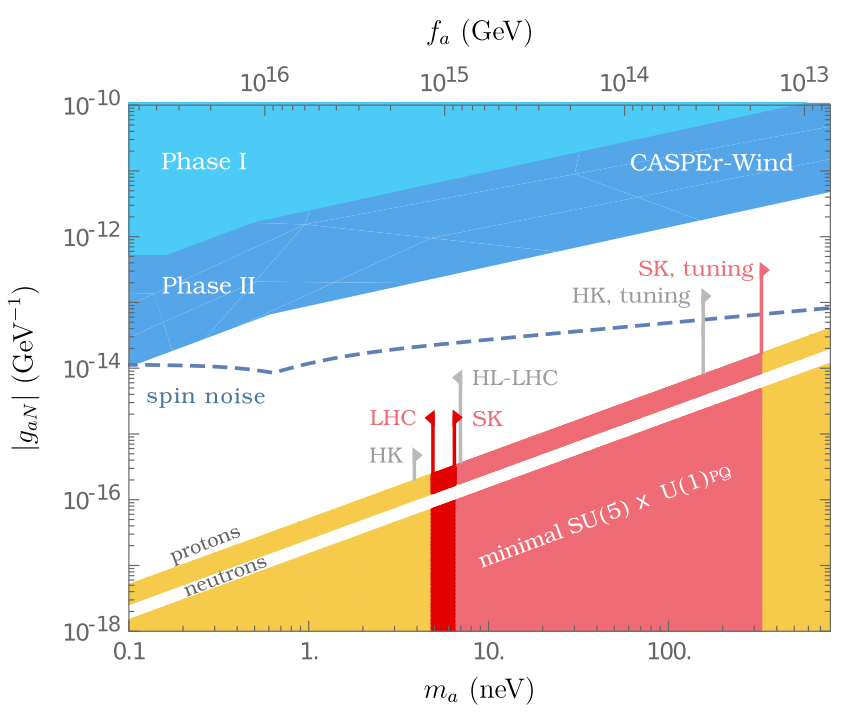

FIG. 4. Axion coupling to the nucleons, $g_{a N}$, versus axion mass $m_{a}$. The blue regions give the projected sensitivities of CASPErWind from Ref. [30].

(via ABRACADABRA and/or CASPEr-Electric) would lead to a direct determination of the GUT scale, possibly discriminating among GUT models, and setting a target for proton decay measurements. Although we exemplified our predictions in the case of the WGG $+24_{F}$ model, it would be interesting to compare axion properties in other minimal extensions of the WGG model which simultaneously address neutrino masses and gauge coupling unification (see e.g., [64-66]), or in realistic $\mathrm{SO}(10)$ models [11].

Finally, the intriguing possibility that the $24_{H}$ field could also be responsible for inflation would make the WGG + $24_{F}$ model a potential candidate for a minimal and predictive GUT-SMASH $[51,67]$ variant aiming at a selfcontained description of particle physics, from the electroweak scale to the Planck scale, and of cosmology, from inflation until today. We leave a detailed investigation of this scenario for future studies.

\section{ACKNOWLEDGMENTS}

We thank Dmitry Budker, Anne Ernst, Mark Goodsell, Maxim Pospelov, Richard Fibonacci Ruiz, Alex Sushkov, and Lindley Winslow for very helpful discussions and communications. C. T. acknowledges support by the Collaborative Research Centre SFB1258 of the Deutsche Forschungsgemeinschaft (DFG). 
[1] K. Abe et al. (Super-Kamiokande Collaboration), Phys. Rev. D 95, 012004 (2017).

[2] K. Abe et al. (Hyper-Kamiokande Working Group), arXiv: 1412.4673.

[3] R. D. Peccei and H. R. Quinn, Phys. Rev. D 16, 1791 (1977).

[4] R. D. Peccei and H. R. Quinn, Phys. Rev. Lett. 38, 1440 (1977).

[5] S. Weinberg, Phys. Rev. Lett. 40, 223 (1978).

[6] F. Wilczek, Phys. Rev. Lett. 40, 279 (1978).

[7] J. Preskill, M. B. Wise, and F. Wilczek, Phys. Lett. 120B, 127 (1983).

[8] L. F. Abbott and P. Sikivie, Phys. Lett. 120B, 133 (1983).

[9] M. Dine and W. Fischler, Phys. Lett. 120B, 137 (1983).

[10] I. G. Irastorza and J. Redondo, Prog. Part. Nucl. Phys. 102, 89 (2018).

[11] A. Ernst, A. Ringwald, and C. Tamarit, J. High Energy Phys. 02 (2018) 103.

[12] D. B. Reiss, Phys. Lett. 109B, 365 (1982).

[13] R. N. Mohapatra and G. Senjanovic, Z. Phys. C 17, 53 (1983).

[14] R. Holman, G. Lazarides, and Q. Shafi, Phys. Rev. D 27, 995 (1983).

[15] B. Bajc, A. Melfo, G. Senjanovic, and F. Vissani, Phys. Rev. D 73, 055001 (2006).

[16] S. Bertolini, L. Di Luzio, and M. Malinsky, Phys. Rev. D 85, 095014 (2012).

[17] G. Altarelli and D. Meloni, J. High Energy Phys. 08 (2013) 021.

[18] K. S. Babu and S. Khan, Phys. Rev. D 92, 075018 (2015).

[19] G. Dvali and A. Vilenkin, Phys. Rev. D 70, 063501 (2004).

[20] G. Dvali, Phys. Rev. D 74, 025018 (2006).

[21] P. W. Graham, D. E. Kaplan, and S. Rajendran, Phys. Rev. Lett. 115, 221801 (2015).

[22] M. B. Wise, H. Georgi, and S. L. Glashow, Phys. Rev. Lett. 47, 402 (1981).

[23] H. P. Nilles and S. Raby, Nucl. Phys. B198, 102 (1982).

[24] H. Georgi and S. L. Glashow, Phys. Rev. Lett. 32, 438 (1974).

[25] B. Bajc and G. Senjanovic, J. High Energy Phys. 08 (2007) 014.

[26] B. Bajc, M. Nemevsek, and G. Senjanovic, Phys. Rev. D 76, 055011 (2007).

[27] L. Di Luzio and L. Mihaila, Phys. Rev. D 87, 115025 (2013).

[28] Y. Kahn, B. R. Safdi, and J. Thaler, Phys. Rev. Lett. 117, 141801 (2016).

[29] D. Budker, P. W. Graham, M. Ledbetter, S. Rajendran, and A. Sushkov, Phys. Rev. X 4, 021030 (2014).

[30] D. F. Jackson Kimball et al., arXiv:1711.08999.

[31] A. R. Zhitnitsky, Yad. Fiz. 31, 497 (1980) [Sov. J. Nucl. Phys. 31, 260 (1980)].

[32] M. Dine, W. Fischler, and M. Srednicki, Phys. Lett. 104B, 199 (1981).

[33] S. M. Boucenna and Q. Shafi, Phys. Rev. D 97, 075012 (2018).
[34] M. Claudson, M. B. Wise, and L. J. Hall, Nucl. Phys. B195, 297 (1982).

[35] P. Nath and P. Fileviez Perez, Phys. Rep. 441, 191 (2007).

[36] A. J. Buras, J. R. Ellis, M. K. Gaillard, and D. V. Nanopoulos, Nucl. Phys. B135, 66 (1978).

[37] F. Wilczek and A. Zee, Phys. Rev. Lett. 43, 1571 (1979).

[38] S. Bertolini, L. Di Luzio, and M. Malinsky, Phys. Rev. D 87, 085020 (2013).

[39] G. Grilli di Cortona, E. Hardy, J. P. Vega, and G. Villadoro, J. High Energy Phys. 01 (2016) 034.

[40] S. Borsanyi et al., Nature (London) 539, 69 (2016).

[41] A. Arhrib, B. Bajc, D. K. Ghosh, T. Han, G.-Y. Huang, I. Puljak, and G. Senjanovic, Phys. Rev. D 82, 053004 (2010).

[42] A. M. Sirunyan et al. (CMS Collaboration), Phys. Rev. Lett. 119, 221802 (2017).

[43] R. Ruiz, J. High Energy Phys. 12 (2015) 165.

[44] Y. Cai, T. Han, T. Li, and R. Ruiz, Front. Phys. 6, 40 (2018).

[45] M. Chabab, M. C. Peyranere, and L. Rahili, arXiv:1805 .00286 .

[46] S. Weinberg, Phys. Lett. 91B, 51 (1980).

[47] L. J. Hall, Nucl. Phys. B178, 75 (1981).

[48] I. Dorsner and P. Fileviez Perez, Phys. Lett. B 625, 88 (2005).

[49] H. Kolesova and M. Malinsky, arXiv:1612.09178.

[50] C. Regis et al. (Super-Kamiokande Collaboration), Phys. Rev. D 86, 012006 (2012).

[51] G. Ballesteros, J. Redondo, A. Ringwald, and C. Tamarit, J. Cosmol. Astropart. Phys. 08 (2017) 001.

[52] M. Tegmark, A. Aguirre, M. Rees, and F. Wilczek, Phys. Rev. D 73, 023505 (2006).

[53] A. D. Linde, Phys. Lett. 158B, 375 (1985).

[54] D. Seckel and M. S. Turner, Phys. Rev. D 32, 3178 (1985).

[55] M. Beltran, J. Garcia-Bellido, and J. Lesgourgues, Phys. Rev. D 75, 103507 (2007).

[56] M. P. Hertzberg, M. Tegmark, and F. Wilczek, Phys. Rev. D 78, 083507 (2008).

[57] J. Hamann, S. Hannestad, G. G. Raffelt, and Y. Y. Y. Wong, J. Cosmol. Astropart. Phys. 06 (2009) 022.

[58] B. L. Spokoiny, Phys. Lett. 147B, 39 (1984).

[59] T. Futamase and K.-i. Maeda, Phys. Rev. D 39, 399 (1989).

[60] R. Fakir and W. G. Unruh, Phys. Rev. D 41, 1783 (1990).

[61] P. W. Graham and S. Rajendran, Phys. Rev. D 88, 035023 (2013).

[62] M. Pospelov and A. Ritz, Nucl. Phys. B573, 177 (2000).

[63] M. Giannotti, I. G. Irastorza, J. Redondo, A. Ringwald, and K. Saikawa, J. Cosmol. Astropart. Phys. 10 (2017) 010.

[64] I. Dorsner and P. Fileviez Perez, Nucl. Phys. B723, 53 (2005).

[65] I. Dorsner, P. Fileviez Perez, and R. Gonzalez Felipe, Nucl. Phys. B747, 312 (2006).

[66] P. Fileviez Perez, A. Gross, and C. Murgui, Phys. Rev. D 98, 035032 (2018).

[67] G. Ballesteros, J. Redondo, A. Ringwald, and C. Tamarit, Phys. Rev. Lett. 118, 071802 (2017). 